\begin{tabular}{|c|c|c|c|}
\hline & НАУЧНО-ТЕХНИЧЕСКИЙ ВЕСТНИК ИН & РМАЦИОННЫХ ТЕХНОЛОГИЙ, МЕХАНИКИ И ОПТИКИ & \\
\hline 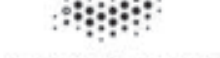 & $\begin{array}{l}\text { январь-февраль } 2018 \quad \text { Том } 18 \text { №1 } \\
\text { SCIENTIFIC AND TECHNICAL JOURNAL O }\end{array}$ & $\begin{array}{l}\text { ISSN 2226-1494 http://ntv.ifmo.rul } \\
\text { IFORMATION TECHNOLOGIES, MECHANICS AND OPTICS }\end{array}$ & 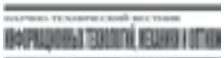 \\
\hline УНИВЕРСИТЕТ ИТМО & January-February 2018 & http://ntv.ifmo.ru/en & \\
\hline
\end{tabular}

\title{
УДК 62.50
}

\section{ПРИМЕНЕНИЕ СХЕМЫ СМИТА В СЛЕДЯЩЕЙ СИСТЕМЕ С ЗАПАЗДЫВАНИЕМ В ОСНОВНОЙ ОБРАТНОЙ СВЯЗИ \\ С.В. Быстров ${ }^{a}$, Н.А. Вундер ${ }^{a}$, А.С. Павлов ${ }^{a}$, М.М. Синетова ${ }^{a}$, А.В. Ушаков}

a Университет ИТМО, Санкт-Петербург, 197101, Российская Федерация

Адрес для переписки: sinetovamadina@gmail.com

Информация о статье

Поступила в редакцию 22.11.17, принята к печати 30.12 .17

doi: 10.17586/2226-1494-2018-18-1-32-42

Язык статьи - русский

Ссылка для цитирования: Быстров С.В., Вундер Н.А., Павлов А.С., Синетова М.М., Ушаков А.В. Применение схемы Смита в следящей системе с запаздыванием в основной обратной связи // Научно-технический вестник информационных технологий, механики и оптики. 2018. Т. 18. № 1. С. 32-42. doi: 10.17586/2226-1494-2018-18-1-32-42

\section{Аннотация}

Предмет исследования. Предметом исследования является системная ситуация, которая возникает при включении непрерывного технического объекта в состав следящей системы, основная обратная связь в которой содержит звено чистого запаздывания. Показано, что для указанной системной ситуации целесообразно строить устройство управления непрерывным техническим объектом в виде последовательного компенсатора. Обнаружено, что, если последовательный компенсатор включается в структуру следящей системы по схеме Смита, то передаточная функция отношения «вход-выход» такой системы не будет содержать передаточную функцию звена чистого запаздывания ни в числителе, ни в знаменателе, тем самым обеспечивается исключение влияния запаздывания в цепи основной обратной связи на поведение системы. Обнаруженное свойство следящей системы с последовательным компенсатором, включенным по схеме Смита, позволяет разработчику последовательного компенсатора использовать алгоритм его синтеза, разработанный для систем управления техническими объектами без запаздывания. Метод. Алгоритмическая база исключения влияния запаздывания в цепи основной обратной связи с помощью последовательного компенсатора, включенного по схеме Смита, основана на использовании метода Мэйсона некасающихся контуров преобразования сигнальных структур и процедуре синтеза последовательного компенсатора как устройства управления для случая непрерывных объектов без запаздывания, опирающихся на свойства типовых полиномиальных динамических моделей. Основные результаты. Разработчик непрерывных следящих систем, в которых основная обратная связь содержит звено чистого запаздывания, получает простой алгоритм синтеза последовательного компенсатора, включение которого по схеме Смита полностью исключает влияние звена чистого запаздывания на поведение системы. Практическая значимость. Полученные результаты имеют практическую ценность для случаев, когда технический объект включается в состав следящей системы, основная обратная связь в которой содержит чистое запаздывание, что особенно важно для реальных технологических процессов. Практическая применимость полученных результатов иллюстрируется компьютерным экспериментом.

\section{Ключевые слова}

технический объект, следящая система, последовательный компенсатор, звено чистого запаздывания в цепи основной обратной связи, схема Смита, правило Мэйсона, алгоритм синтеза последовательного компенсатора

Благодарности

Работа выполнена при поддержке Правительства Российской Федерации (Грант 074-U01), Министерства образования и науки Российской Федерации (Проект 14. Z50.31.0031), гранта Президента Российской Федерации №14.Y31.16.9281-НШ.

\section{SMITH SCHEME APPLICATION IN THE SERVO SYSTEM WITH DELAY IN THE MAIN FEEDBACK}

\section{S.V. Bystrova , N.A. Vunder ${ }^{a}$, A.S. Pavlov ${ }^{a}$, M.M. Sinetova ${ }^{a}$, A.V. Ushakov}

${ }^{a}$ ITMO University, Saint Petersburg, 197101, Russian Federation

Corresponding author: sinetovamadina@gmail.com

\section{Article info}

Received 22.11.17, accepted 30.12.17

doi: 10.17586/2226-1494-2018-18-1-32-42

Article in Russian 
For citation: Bystrov S.V., Vunder N.A., Pavlov A.S., Sinetova M.M., Ushakov A.V. Smith scheme application in the servo system with delay in the main feedback. Scientific and Technical Journal of Information Technologies, Mechanics and Optics, 2018, vol. 18, no. 1, pp. 32-42 (in Russian). doi: 10.17586/2226-1494-2018-18-1-32-42

\section{Abstract}

Subject of Research. The subject of the study is the system situation that arises when a continuous plant is included in the system, the main feedback in which contains the link of pure delay. It is shown that for this system situation it is advisable to design a control device for continuous plant in the form of a consecutive compensator. It is found that if the consecutive compensator is included in the structure of the system according to the Smith scheme, then the transfer function of the inputoutput ratio of such system will not contain the transfer function of the pure delay link neither in the numerator, nor in the denominator, therefore the delay in the main feedback does not affect the system behavior. This property of the system with a consecutive compensator included in the Smith scheme enables the developer to use an algorithm of its synthesis created for plant control systems without delay. Method. The algorithmic basis is based on the use of the Mason method of nontangential circuits for the transformation of signal structures and the procedure for the synthesis of a consecutive compensator, as a device for controlling continuous plants without delay. The method is based on the properties of typical polynomial models. Main Results. The developer of continuous systems with main feedback with delay obtains a simple algorithm for the synthesis of a consecutive compensator included in the Smith scheme, which completely excludes the effect of the pure delay link on the system behavior. Practical Relevance. The obtained results are of practical value for cases when a plant is included in the system with main feedback containing a pure delay. This is especially important for real technological processes. Practical application of the obtained results is illustrated by computer experiment.

\section{Keywords}

continuous plant, servo system, consecutive compensator, pure delay link in the main feedback, Smith scheme, Mason rule, consecutive compensator synthesis algorithm

Acknowledgements

This work was supported by the Government of the Russian Federation, Grant 074-U01; the Ministry of Education and Science of the Russian Federation, Project 14.Z50.31.0031; the Russian Federation President Grant No.14.Y31.16.9281-HШ.

\section{Введение. Постановка задачи}

В настоящее время в практике разработки устройств управления непрерывными техническими объектами сложилось два алгоритмических подхода.

Первый подход, который в основном разрабатывается при использовании векторно-матричных модельных представлений пространства состояний, основан на концепции векторно-матричного подобия процессов, происходящих в проектируемой следящей системе, процессам, происходящим в некоторой эталонной модели желаемого поведения, изначально задаваемой передаточной функцией типовой полиномиальной динамической модели (ТПДМ) [1-10], процедурно преобразуемой к векторно-матричной форме в процессе синтеза. Этот подход, реализуемый в форме модального управления, позволяет синтезировать системы в два этапа. На первом этапе синтезируется устройство управления в виде композиции прямых связей по задающему воздействию и обратных связей по вектору состояния. На втором этапе в силу того, что компоненты вектора состояния не являются полностью измеримыми, синтезируется алгоритм формирования оценок компонентов вектора состояния, именуемого обычно наблюдающим устройством. Такой подход хорошо зарекомендовал себя в задачах локального управления техническими объектами без запаздывания, так как звено чистого запаздывания не может быть представлено инструментарием метода пространства состояний.

Второй подход в практике разработки устройств управления непрерывными техническими объектами основан на использовании аппарата передаточной функции и концепции последовательной компенсации нежелаемых полюсов технического объекта нулями передаточной функции устройств управления, которое в данном случае именуется последовательным компенсатором (ПК). Для синтеза последовательного компенсатора необходимо наличие передаточной функции типовой полиномиальной динамической модели «вход-выход», из которой извлекается передаточная функция прямой ветви системы с единичной отрицательной обратной связью. Заметным преимуществом этого подхода является то, что для реализации ПК достаточно информации только о рассогласовании выходной переменной и задающего воздействия. Более того, в отличие от метода пространства состояний, аппарат передаточной функции в случае появления в структуре системы звена чистого запаздывания (ЗЧЗ) позволяет представить его передаточной функцией.

В последнее время алгоритмическая практика синтеза последовательного компенсатора в задачах управления обогатилась схемой Смита включения ПК в состав системы управления объектом, обладающим запаздыванием в канале управления [4-8]. Схема Смита включения ПК сняла проблему влияния запаздывания на устойчивость системы с ПК, но породила проблемы, связанные с длительностью переходного процесса и качественными показателями в установившемся режиме при конечномерных внешних воздействиях $[11,12]$.

В настоящей работе ставится и решается задача исключения влияния запаздывания в цепи основной обратной связи с помощью ПК, включенного по схеме Смита. 


\section{Предварительные сведения. Алгоритм синтеза последовательного компенсатора для случая отсутствия запаздывания в цепи основной обратной связи}

В настоящее время синтез ПК [13] для управления техническим объектом, в составе передаточной функции которого отсутствует ЗЧЗ как на входе, так и на выходе, может быть осуществлен с помощью следующего алгоритма.

\section{Алгоритм 1.}

1. Задать технические требования к показателям качества проектируемой следящей системы в переходном режиме в виде допустимой величины перерегулирования $\sigma_{\partial}$ и допустимой длительности переходного процесса $t_{\text {пә }}$, а также в установившемся режиме в виде необходимой величины добротности по скорости $D_{1 \partial}$ или величины полосы пропускания $\Delta \omega_{\delta ə}$ на уровне $\delta_{\partial}$ относительной частотной ошибки $\delta(\omega)$.

2. Задать аналитическое представление передаточной функции $W_{\text {то }}(s)$ управляемого технического объекта (ТО).

3. Задать передаточную функцию $\Phi\left(s, \omega_{0}\right)$ типовой полиномиальной динамической модели [1-3] отношения «вход-выход» проектируемой следящей системы в форме, параметризованной характеристической частотой $\omega_{0}$ :

$$
\Phi\left(s, \omega_{0}\right)=\frac{y(s)}{g(s)}=\frac{v_{n} \omega_{0}^{n}}{s^{n}+\sum_{i=1}^{n} v_{i} \omega_{0}^{i} s^{n-i}}=\frac{v_{n} \omega_{0}^{n}}{D\left(s, \omega_{0}\right)}
$$

где $y(s)$ и $g(s)$ - соответственно преобразования Лапласа выходной переменной $y(t)$ системы и задающего воздействия $g(t)$, коэффициенты $v_{i}(i=\overline{1, n})$ определяют характер размещения корней полинома знаменателя (ПЗ) $D\left(s, \omega_{0}\right)$ передаточной функции на комплексной плоскости.

4. На основании аналитических связей численных величин показателей качества проектируемой следящей системы с характеристической частотой $\omega_{0}$ ее передаточной функции [3] оценить ее значения в силу правила

$$
\omega_{0}=\max \left\{\omega_{0}=\arg \left(t_{n}\left(\omega_{0}\right) \leq t_{\Pi \partial}\right), \omega_{0}=\arg \left(D_{1}\left(\omega_{0}\right) \geq D_{1 \partial}\right), \omega_{0}=\arg \left(\Delta \omega_{\delta}\left(\omega_{0}\right) \geq \Delta \omega_{\delta \partial}\right)\right\} .
$$

5. С использованием результата (2) сконструировать передаточную функцию $W\left(s, \omega_{0}\right)$ прямой ветви отношения «ошибка-выход» проектируемой следящей системы, которая в силу (1) принимает вид

$$
W\left(s, \omega_{0}\right)=\frac{y(s)}{\varepsilon(s)}=\frac{\Phi\left(s, \omega_{0}\right)}{1-\Phi\left(s, \omega_{0}\right)}=\frac{v_{n} \omega_{0}^{n}}{s^{n}+v_{1} \omega_{0} s^{n-1}+\ldots+v_{n-1} \omega_{0}^{n-1} s} .
$$

где $\varepsilon(s)$ - преобразование Лапласа ошибки $\varepsilon(t)=g(t)-y(t)$ воспроизведения следящей системой задающего воздействия.

6. Используя (3), сформировать передаточную функцию $W_{\text {пк }}\left(s, \omega_{0}\right)$ ПК с помощью аналитического соотношения

$$
W_{\text {пк }}\left(s, \omega_{0}\right)=W\left(s, \omega_{0}\right) / W_{\text {то }}(s) .
$$

7. Проверить передаточную функцию ПК $W_{\text {Пк }}\left(s, \omega_{0}\right)$ на физическую реализуемость контролем ее относительной степени путем сравнения степеней полиномов числителя и знаменателя. Если она неотрицательна, то перейти к п. 8 алгоритма, иначе к п. 3 с целью увеличения размерности $n$ типовой полиномиальной динамической модели.

8. Осуществить комплексное компьютерное исследование системы с спроектированным ПК в среде MATLAB. В случае удовлетворения показателей качества спроектированной следящей системы требований п. 1 перейти к п. 9, в противном случае перейти к п. 4 с целью изменения значения характеристической частоты.

9. С помощью процедуры с2d в среде MATLAB с интервалом дискретности $\Delta t \leq \frac{\pi}{\left(\frac{v_{n}}{\delta_{y}}\right)^{1 / n} \omega_{0}}$ при

$\delta_{y} \in[0,01-0,05]$ осуществить переход от непрерывного представления с передаточной функцией 
$W_{\text {пк }}\left(s, \omega_{0}\right)$ ПК к его дискретному представлению $W_{\text {пк }}\left(z, \omega_{0}\right)$ с целью программирования микроконтроллера управляющего устройства.

Дадим комментарий к приведенному алгоритму. Он касается в основном п.п. 3, 4 и 9. Передаточная функция $\Phi\left(s, \omega_{0}\right)(1)$ типовой полиномиальной динамической модели в п. 3 , которая в задаче синтеза системы управления является желаемой передаточной функцией замкнутой следящей системы, параметризованной характеристической частотой $\omega_{0}$, характеризуется тем, что ее коэффициенты $v_{i}$ определяют тип размещения корней $D\left(s, \omega_{0}\right)$ ПЗ $\Phi\left(s, \omega_{0}\right)$, а $\omega_{0}$ определяет размер области локализации этого размещения. Основным преимуществом представления передаточной функции $\Phi\left(s, \omega_{0}\right)$ в форме (1) является то, что ее использование при фиксированных $v_{i}$ сводит задачу синтеза к однопараметрической задаче поиска значения характеристической частоты $\omega_{0}$, от которой зависят длительность переходного процесса, добротность по скорости, частота среза, полосы пропускания на заданном уровне амплитудных частотных характеристик отношений «вход-выход» и «вход-ошибка». В свою очередь, от коэффициентов $v_{i}$ зависят величина перерегулирования, показатель колебательности и величина запаса устойчивости по фазе.

В случае использования при синтезе следящей системы ТПДМ с размещением Ньютона корней полинома $D\left(s, \omega_{0}\right)$ компоненты $v_{i}$ его коэффициентов определяются соотношениями

$$
v_{i}=C_{n}^{i}(i=\overline{1, n}),
$$

а при синтезе следящей системы с круговым размещением Баттерворта корней полинома $D\left(s, \omega_{0}\right)$, его коэффициенты $v_{i}$ определяются соотношениями

$$
v_{1}=\frac{1}{\sin (\pi /(2 n))}, v_{i}=\frac{v_{i-1} \cos ((i-1) \pi /(2 n))}{\sin (i \pi /(2 n))},(i=\overline{1, n-1}), v_{n}=1 .
$$

Следуя [3], приведем в табличной форме аналитические представления показателей качества

\begin{tabular}{|c|c|c|c|c|c|c|c|}
\hline \multirow{2}{*}{$\begin{array}{c}\text { Аналитическое представление } \\
\text { полинома знаменателя } \\
D\left(s, \omega_{0}\right)\end{array}$} & \multirow[b]{2}{*}{$\sigma$} & \multirow[b]{2}{*}{$t_{\Pi \Pi}\left(\omega_{0}\right)$} & \multirow[b]{2}{*}{$\Delta \varphi$} & \multirow[b]{2}{*}{$\omega_{c}\left(\omega_{0}\right)$} & \multirow[b]{2}{*}{$D_{1}\left(\omega_{0}\right)$} & \multicolumn{2}{|c|}{$\begin{array}{c}\text { Полоса пропускания } \\
\Delta \omega / \omega_{0}\end{array}$} \\
\hline & & & & & & 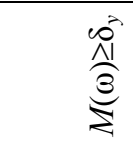 & $\frac{\overbrace{0}^{\omega}}{V_{0}^{\infty}}$ \\
\hline 1 & 2 & 3 & 4 & 5 & 6 & 7 & 8 \\
\hline$s^{n}+\sum_{i=1}^{n} v_{i} \omega_{0}^{i} s^{n-i}$ & $\sigma^{*}$ & $\frac{t_{\Pi}^{*}}{\omega_{0}}$ & $\Delta^{*} \varphi\left(v_{i}\right)$ & $\frac{v_{n} \omega_{0}}{v_{n-1}}$ & $\frac{v_{n} \omega_{0}}{v_{n-1}}$ & $\left(\frac{v_{n}}{\delta_{y}}\right)^{1 / n}$ & $\frac{\delta_{\varepsilon} v_{n}}{v_{n-1}}$ \\
\hline
\end{tabular}
ТПДМ (табл. 1).

Таблица 1. Общесистемные показатели качества ТПДМ

В табл. 1 в графе 3 указано время переходного процесса, в графе 5 - частота среза, в графе 6 - добротность по скорости, в графах 7-8 указаны полосы пропускания системы на различных уровнях амплитудной частотной характеристики $M(\omega)$ отношения «вход-выход» ТПДМ и амплитудной частотной характеристики $\delta(\omega)$, в графе 2 - величина перерегулирования, в графе 4 - запас устойчивости по фазе. Показатели качества ТПДМ, приведенные в графах 2 и 4, зависят только от коэффициентов $v_{i}$ полинома $D\left(s, \omega_{0}\right)$, показатели качества ТПДМ в приведенных остальных графах зависят как от коэффициентов $v_{i}$, так и от характеристической частоты $\omega_{0}$.

В табл. 2 приведены аналитические представления запаса устойчивости по фазе $\Delta \varphi$ для различных порядков ТПДМ $n$.

В табл. 3 представлены показатели качества переходной характеристики ТПДМ различных порядков. 


\begin{tabular}{|c|l|}
\hline $\begin{array}{c}\text { Порядок ТПДМ } \\
n\end{array}$ & \multicolumn{1}{|c|}{ Аналитическое представление запаса устойчивости по фазе $\Delta \varphi=\Delta \varphi\left(v_{i} ; i=\overline{1, n}\right)$} \\
\hline$n=1$ & $\Delta \varphi=\pi / 2+\operatorname{arctg}\left(0 / v_{1}\right)=\pi / 2$ \\
\hline$n=2$ & $\Delta \varphi=\pi / 2-\operatorname{arctg}\left(v_{2} / v_{1}^{2}\right)$ \\
\hline$n=3$ & $\Delta \varphi=\pi / 2-\operatorname{arctg}\left(v_{1}\left(v_{3} / v_{2}\right) /\left(v_{2}-\left(v_{3} / v_{2}\right)^{2}\right)\right)$ \\
\hline$n=4$ & $\Delta \varphi=\pi / 2-\operatorname{arctg}\left(\left(v_{4} / v_{3}\right)\left(v_{2}-\left(v_{4} / v_{3}\right)^{2}\right) /\left(v_{3}-v_{1}\left(v_{4} / v_{3}\right)^{2}\right)\right)$ \\
\hline$n=5$ & $\Delta \varphi=\pi / 2-\operatorname{arctg}\left(\left(v_{5} / v_{4}\right)\left(v_{3}-v_{1}\left(v_{5} / v_{4}\right)^{2}\right) /\left(v_{4}-v_{2}\left(v_{5} / v_{4}\right)^{2}+\left(v_{5} / v_{4}\right)^{4}\right)\right)$ \\
\hline
\end{tabular}

Таблица 2. Аналитические представления запасов устойчивости $\Delta^{*} \varphi\left(v_{i}\right)$ по фазе ТПДМ как функции ее порядка

\begin{tabular}{|c|c|c|c|c|c|}
\hline \multirow{2}{*}{$\begin{array}{c}\text { № } \\
\text { п.п. }\end{array}$} & $\begin{array}{c}\text { Порядок ТПдМ } \\
n\end{array}$ & \multicolumn{2}{|c|}{$\begin{array}{c}\text { Перерегулирование } \\
\sigma^{*}, \%\end{array}$} & \multicolumn{2}{c|}{ Длительность переходного процесса $t_{\Pi}{ }^{*}$} \\
\cline { 2 - 6 } & $n=1$ & 0 & 0 & $v_{i}$ вида (5) & $v_{i}$ вида (6) \\
\hline 1 & $v_{i}$ вида (5) & $v_{i}$ вида (6) & 3 & 3 \\
\hline 2 & $n=2$ & 0 & 5 & 4,75 & 4,5 \\
\hline 3 & $n=3$ & 0 & 9 & 6,3 & 7 \\
\hline 4 & $n=4$ & 0 & 11 & 7,8 & 8 \\
\hline 5 & $n=5$ & 0 & 13 & 9,15 & 7 \\
\hline
\end{tabular}

Таблица 3. Показатели качества переходной характеристики ТПДМ как функции ее порядка и представления коэффициентов $v_{i}$

\section{Базовая структура схемы Смита включения последовательного компенсатора в цепь управления техническим объектом с запаздыванием}

Базовая структура схемы Смита включения последовательного компенсатора с передаточной функцией (4) в основном применяется $[4,5,13]$ для случая, когда ТО характеризуется наличием чистого запаздывания в цепи управления и описывается передаточной функцией

$$
W_{\text {то }}(s, \tau)=W_{\text {то }}(s) e^{-\tau s},
$$

где $\tau$ - величина чистого запаздывания.

Данная схема для объекта с передаточной функцией (7) приведена на рис. 1.

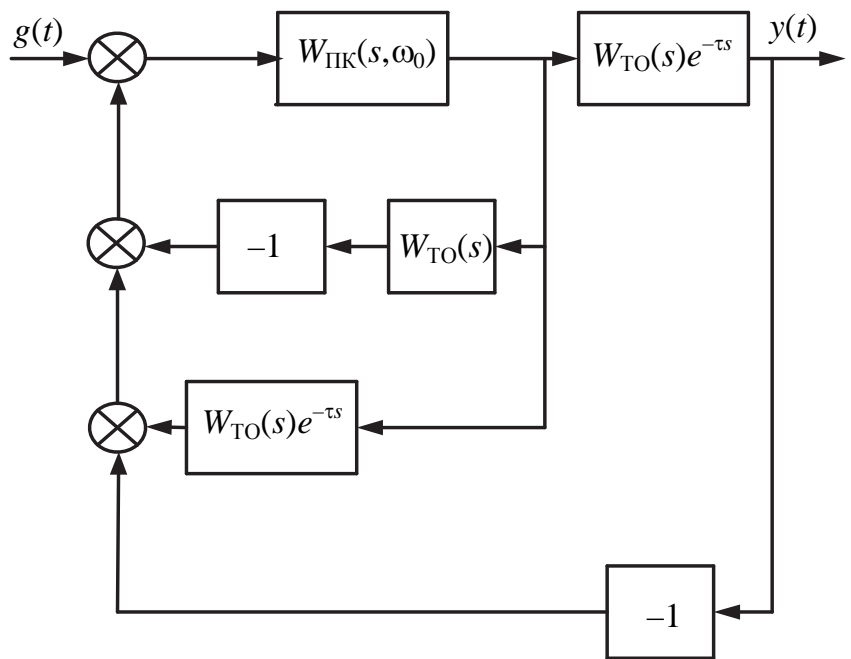

Рис. 1. Схема включения последовательного компенсатора по схеме О. Смита

Из рис. 1 видно, что схема Смита включения ПК характеризуется наличием трех контуров. Первый контур, в который вложены все остальные, описывается передаточной функцией $W_{1}\left(s, \omega_{0}, \tau\right)=-W_{\text {пк }}\left(s, \omega_{0}\right) W_{\text {то }}(s) e^{-\tau s}$. Второй контур описывается передаточной функцией 
$W_{2}\left(s, \omega_{0}, \tau\right)=W_{\text {пк }}\left(s, \omega_{0}\right) W_{\text {то }}(s) e^{-\tau s}$. Третий контур описывается передаточной функцией $W_{3}\left(s, \omega_{0}\right)=-W_{\text {пк }}\left(s, \omega_{0}\right) W_{\text {то }}(s)$. Прямая ветвь (ПВ), связывающая вход, на который подается входное воздействие $g(t)$, с выходом системы, на котором формируется выходная переменная $y(t)$, описывается передаточной функцией $W_{\text {Пв }}\left(s, \omega_{0}, \tau\right)=W_{\text {пк }}\left(s, \omega_{0}\right) W_{\text {то }}(s) e^{-\tau s}$. Если к структурной схеме рис. 1 применить правило Мейсона некасающихся контуров $[14,15]$, то в соответствии с ним передаточная функция схемы рис. 1 получит представление

$$
\begin{aligned}
& \Phi\left(s, \omega_{0}, \tau\right)=\frac{W_{\text {Пв }}\left(s, \omega_{0}, \tau\right)}{1-W_{3}\left(s, \omega_{0}\right)-W_{2}\left(s, \omega_{0}, \tau\right)-W_{1}\left(s, \omega_{0}, \tau\right)}= \\
& =\frac{W_{\text {ПК }}\left(s, \omega_{0}\right) W_{\text {тО }}(s) e^{-\tau s}}{1+W_{\text {пК }}\left(s, \omega_{0}\right) W_{\text {ТО }}(s)-W_{\text {ПК }}\left(s, \omega_{0}\right) W_{\text {ТО }}(s) e^{-\tau s}+W_{\text {пК }}\left(s, \omega_{0}\right) W_{\text {ТО }}(s) e^{-\tau s}}= \\
& =\frac{W_{\text {ПК }}\left(s, \omega_{0}\right) W_{\text {ТО }}(s) e^{-\tau s}}{1+W_{\text {ПК }}\left(s, \omega_{0}\right) W_{\text {ТО }}(s)}=\Phi\left(s, \omega_{0}\right) e^{-\tau s} .
\end{aligned}
$$

Нетрудно видеть, что схема Смита включения последовательного компенсатора решает главную задачу - исключение влияния ЗЧЗ на устойчивость следящей системы путем выноса ЗЧЗ за пределы главного контура системы. Это достигается в схеме Смита с помощью процедуры сигнальной компенсации. Следует отметить, что $3 Ч 3 e^{-\tau s}$ и передаточная функция $W_{\text {то }}(s)$ в первом контуре в составе его передаточной функции $W_{1}\left(s, \omega_{0}, \tau\right)$ обладают реальными параметрами технического объекта, в то время как те же звенья второго и третьего контуров в составе передаточных функций $W_{2}\left(s, \omega_{0}, \tau\right)$ и $W_{3}\left(s, \omega_{0}\right)$ обладают параметрами, реализуемыми техническими средствами устройства управления, которое построено на агрегировании первого и второго контуров и ПК. Анализ параметрической чувствительности траекторий следящей системы (рис. 1) с помощью аппарата теории чувствительности в рамках функций чувствительности первого порядка обнаружил [12] робастность схемы Смита при малой неопределенности чистого запаздывания в техническом объекте. Дополнительно о схеме Смита нужно сказать, что наличие ЗЧЗ в ее передаточной функции «вход-выход» (8) заметно влияет на показатели качества следящей системы в установившемся режиме, так что добротность по скорости системы, сформированной по схеме Смита, не превышает $1 / \tau$.

\section{Основной результат. Исключение влияния запаздывания в цепи основной обратной связи с помощью последовательного компенсатора, включенного по схеме Смита}

Схема Смита включения последовательного компенсатора в состав проектируемой следящей системы обнаружила дополнительные системные возможности. Рассмотрим случай системной ситуации, когда запаздывание существует в основной обратной связи системы, в состав которой включен технический объект без запаздывания. Этому случаю соответствует структурная схема системы, приведенная на рис. 2, построенная на основе традиционного включения ПК в состав системы в случае наличия запаздывания в цепи основной обратной связи.

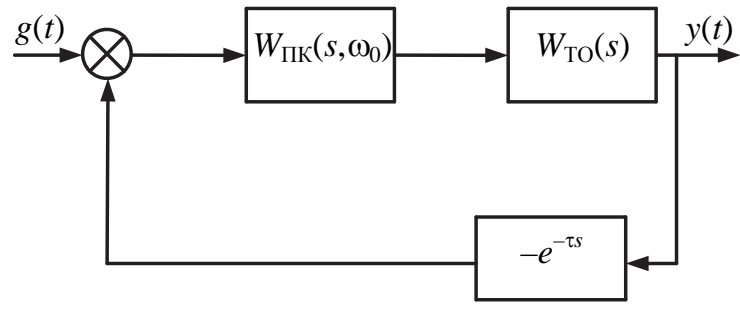

Рис. 2. Схема традиционного включения последовательного компенсатора в состав следящей системь в случае наличия запаздывания в цепи основной обратной связи

Передаточная функция отношения «вход-выход» приведенной на рис. 2 схемы имеет вид

$$
\Phi\left(s, \omega_{0}, \tau\right)=\frac{W_{\text {ПК }}\left(s, \omega_{0}\right) W_{\text {то }}(s)}{1+W_{\text {ПК }}\left(s, \omega_{0}\right) W_{\text {ТО }}(s) e^{-\tau s}}=\frac{W\left(s, \omega_{0}\right)}{1+W\left(s, \omega_{0}\right) e^{-\tau s}} .
$$

Нетрудно видеть, что приведенные во втором разделе предварительные сведения позволяют оценить ограничения, накладываемые на пару параметров « $\tau-\omega_{0}$ » из условия равенства нулю запаса устойчивости системы (9) по фазе:

$$
\Delta \varphi\left\{W\left(j \omega, \omega_{0}\right)\right\}-\tau \omega_{c}=0 .
$$


Здесь $\Delta \varphi\left\{W\left(j \omega, \omega_{0}\right)\right\}$ - запас устойчивости по фазе используемой ТПДМ, приведенный в табл. 2, $\omega_{c}$ - частота среза, аналитическое представление $\omega_{c}\left(\omega_{0}\right)$ которой в силу табл. 1 задается соотношением

$$
\omega_{\mathrm{c}}=\frac{v_{n} \omega_{0}}{v_{n-1}} .
$$

Два полученные выше соотношения позволяют записать:

$$
\tau \omega_{0}=\frac{v_{n-1}}{v_{n}} \Delta \varphi\left\{W\left(j \omega, \omega_{0}\right)\right\},
$$

т.е. устойчивость системы рис. 2 гарантируется при выполнении неравенства

$$
\tau \omega_{0}<\frac{v_{n-1}}{v_{n}} \Delta \varphi\left\{W\left(j \omega, \omega_{0}\right)\right\} .
$$

Очевидно, условие (10) является сильным ограничением на выбор значений характеристической частоты при заданном значении запаздывания при обеспечении устойчивости системы с передаточной функцией (9), оно оказывается еще более жестким при обеспечении требований к качеству управляемых процессов в следящей системе в установившемся режиме, что делает практическое использование системы, реализуемой по схеме рис. 2, мало полезным.

Поставим задачу провести модификацию схемы Смита включения последовательного компенсатора с целью вывести из передаточной функции (9) передаточную функцию звена чистого запаздывания $e^{-\tau s}$, чтобы следящая система с запаздыванием в канале обратной связи описывалась бы передаточной функцией вида

$$
\Phi\left(s, \omega_{0}\right)=\frac{W_{\text {ПК }}\left(s, \omega_{0}\right) W_{\text {тО }}(s)}{1+W_{\text {пК }}\left(s, \omega_{0}\right) W_{\text {ТО }}(s)} .
$$

Утверждение. Модифицированная схема Смита включения последовательного компенсатора наделяет передаточную функцию следящей системы «вход-выход» при наличии запаздывания в цепи основной обратной связи представлением (11).

Доказательство. Доказательство утверждения строится на формировании модифицированной базовой структуры схемы Смита рис. 1 включения последовательного компенсатора в состав следящей системы и применении к ней правила Мейсона некасающихся контуров [14, 15]. Модифицированная базовая структура схемы Смита включения последовательного компенсатора в состав следящей системы при наличии запаздывания в цепи основной обратной связи представлена на рис. 3.

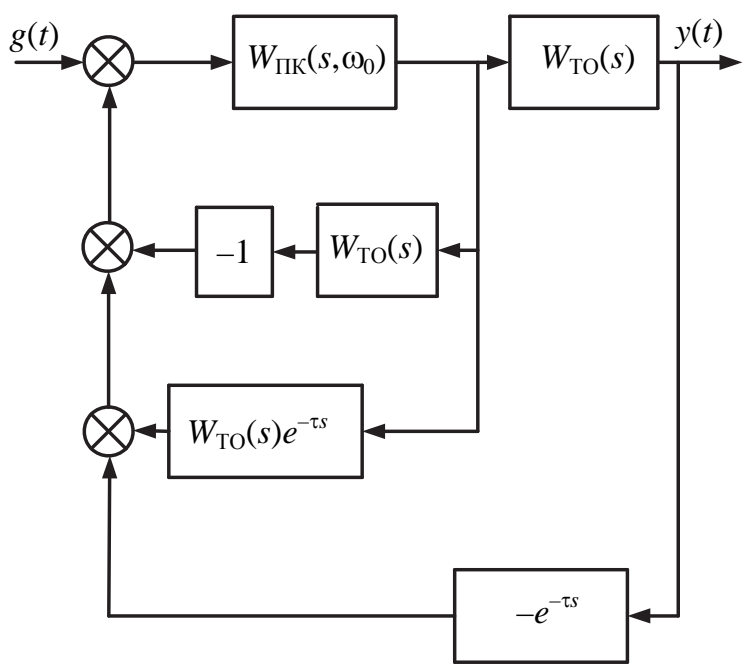

Рис. 3. Модифицированная схема О. Смита включения последовательного компенсатора в состав следящей системы с запаздыванием в основной обратной связи

Если к структурной схеме рис. 3 применить правило Мейсона некасающихся контуров [14, 15], то в соответствии с ним передаточная функция следящей системы рис. 3 получит представление

$$
\begin{aligned}
& \Phi\left(s, \omega_{0}, \tau\right)=\frac{W_{\text {пК }}\left(s, \omega_{0}\right) W_{\text {ТО }}(s)}{1+W_{\text {ПК }}\left(s, \omega_{0}\right) W_{\text {ТО }}(s)-W_{\text {ПК }}\left(s, \omega_{0}\right) W_{\text {ТО }}(s) e^{-\tau s}+W_{\text {ПК }}\left(s, \omega_{0}\right) W_{\text {ТО }}(s) e^{-\tau s}}= \\
& =\frac{W_{\text {ПК }}\left(s, \omega_{0}\right) W_{\text {ТО }}(s)}{1+W_{\text {ПК }}\left(s, \omega_{0}\right) W_{\text {ТО }}(s)}=\Phi\left(s, \omega_{0}\right) .
\end{aligned}
$$


Утверждение доказано.

Нетрудно видеть, что алгоритм синтеза последовательного компенсатора для системной ситуации, когда в цепи основной обратной связи следящей системы существует запаздывание, при его включении по модифицированной схеме Смита, приведенной на рис. 3, свелся к алгоритму 1 синтеза последовательного компенсатора для случая отсутствия запаздывания в прямой цепи и цепи основной обратной связи с точностью до его модификации, начиная с п. 8, в форме:

8. Включить разработанный последовательный компенсатор с передаточной функцией $W_{\text {пк }}\left(s, \omega_{0}\right)$ в состав модифицированной схемы Смита рис. 3.

9. Выполнить пункт 8 алгоритма 1.

10. Выполнить пункт 9 алгоритма 1.

\section{Иллюстративный пример}

1. Зададим технические требования к показателям качества проектируемой следящей системы в переходном режиме в виде допустимой величины перерегулирования $\sigma_{\partial}=0 \%$ и допустимой длительности переходного процесса $t_{\text {пә }} \leq 0,63 \mathrm{c}$, а также в установившемся режиме в виде необходимой величины добротности по скорости $D_{10} \geq 50 \mathrm{c}^{-1}$.

2. 2.1. Зададим аналитическое представление передаточной функции $W_{\text {то }}(s)$ управляемого ТО.

$$
W_{\text {то }}(s)=\frac{1}{(s+1)(s+2) s} .
$$

2.2.Охарактеризуем запаздывание в цепи основной обратной связи величиной $\tau=0,3 \mathrm{c}$.

3. Зададим передаточную функцию $\Phi\left(s, \omega_{0}\right)$ типовой полиномиальной динамической модели [1-3] отношения «вход-выход» проектируемой следящей системы, параметризованную характеристической частотой $\omega_{0}$, в силу $\sigma_{\partial}=0 \%$ с биномиальным распределением Ньютона корней ее ПЗ степени, равной степени ПЗ передаточной функции технического объекта так, что она примет вид

$$
\Phi\left(s, \omega_{0}\right)=\frac{\omega_{0}^{3}}{s^{3}+3 \omega_{0} s^{2}+3 \omega_{0}^{2} s+\omega_{0}^{3}} .
$$

4. На основании аналитических связей величин показателей качества проектируемой следящей системы с характеристической частотой $\omega_{0}$ ее передаточной функции [3], представленных в табл. 1 и табл. 3, оценим ее значения в силу правила

$$
\omega_{0}=\max \left\{\omega_{0}=\arg \left(t_{\Pi} \omega_{0}=0,63 \omega_{0} \leq 6,3\right)=10, \omega_{0}=\arg \left(D_{1}\left(\omega_{0}\right)=\left.\frac{\omega_{0}}{n}\right|_{n=3} \geq 50 \mathrm{c}^{-1}\right)=150\right\}=150 \mathrm{c}^{-1},
$$

что доставит переходным процессам сходимость со временем $t_{\text {П }}=6,3 / 150=0,042 \mathrm{c}$.

5. С использованием результата (2) сконструируем передаточную функцию $W\left(s, \omega_{0}\right)$ прямой ветви отношения «ошибка-выход» проектируемой следящей системы, которая в силу (12) принимает вид

$$
W\left(s, \omega_{0}\right)=\frac{y(s)}{\varepsilon(s)}=\frac{\Phi\left(s, \omega_{0}\right)}{1-\Phi\left(s, \omega_{0}\right)}=\frac{150^{3}}{\left(s^{2}+450 s+3 \cdot 150^{2}\right) s},
$$

где $\varepsilon(s)$ - преобразование Лапласа ошибки $\varepsilon(t)=g(t)-y(t)$ воспроизведения следящей системой задающего воздействия $g(t)$.

6. Сформируем передаточную функцию $W_{\text {пк }}\left(s, \omega_{0}\right)$ последовательного компенсатора с помощью аналитического соотношения

$$
W_{\text {пК }}\left(s, \omega_{0}\right)=W\left(s, \omega_{0}\right) / W_{\text {то }}(s)=\frac{150^{3}(s+1)(s+2)}{s^{2}+450 s+3 \cdot 150^{2}} .
$$

7. Проведем проверку последовательного компенсатора на физическую реализуемость путем контроля относительной степени его передаточной функции $W_{\text {пк }}\left(s, \omega_{0}\right)$, которая является неотрицательной, а потому последовательный компенсатор оказался реализуемым.

8. Включаем разработанный последовательный компенсатор с передаточной функцией $W_{\text {пк }}\left(s, \omega_{0}\right)$ в состав модифицированной схемы Смита рис. 3 , в результате чего получаем схему (рис. 4). 


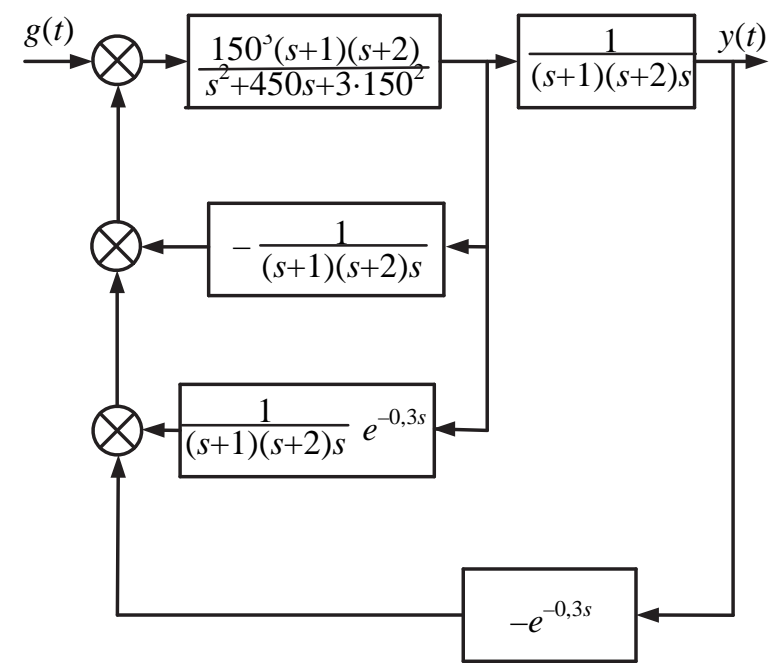

Рис. 4. Модифицированная схема О. Смита включения последовательного компенсатора в иллюстративном примере

9. Комплексное компьютерное исследование следящей системы с разработанным последовательным компенсатором, включенным в нее по схеме рис. 4, проведем в среде MATLAB при внешнем воздействии полиномиального вида $g(t)=g_{0}+\dot{g}_{0} t$, где $g_{0}=0,2, \dot{g}_{0}=-2 \mathrm{c}^{-1}$. Для подтверждения полученных результатов проведем исследование следящей системы для трех значений запаздывания $(\tau=0,06 \mathrm{c}, \tau=0,3 \mathrm{c}, \tau=1,5 \mathrm{c})$ в цепи основной обратной связи. Крайние значения $\tau$ отличаются от первоначального $\tau=0,3$ в в пять раз соответственно в сторону уменьшения и увеличения. Результаты комплексного компьютерного исследования рис. 5 представлены в виде кривых задающего воздействия $g(t)$, выхода $y(t)$ и ошибки $\varepsilon(t)$. Из кривых видно, что длительность переходных процессов $t_{\Pi}=0,042 \mathrm{c}<t_{\Pi \partial}=0,63 \mathrm{c}, \quad$ перерегулирование $\sigma=\sigma_{\partial}=0 \%, \quad$ добротность $D_{1}=\frac{\dot{g}_{0}}{\varepsilon_{\text {уст }}}=\frac{-2 \mathrm{c}^{-1}}{-0,04}=50 \mathrm{c}^{-1} \geq D_{10}=50 \mathrm{c}^{-1}$ удовлетворяют предъявленным к следящей системе требованиям, при этом все кривые процессов для случаев $\tau=0,06 \mathrm{c}, \tau=0,3 \mathrm{c}, \tau=1,5 \mathrm{c}$ оказались идентичными.

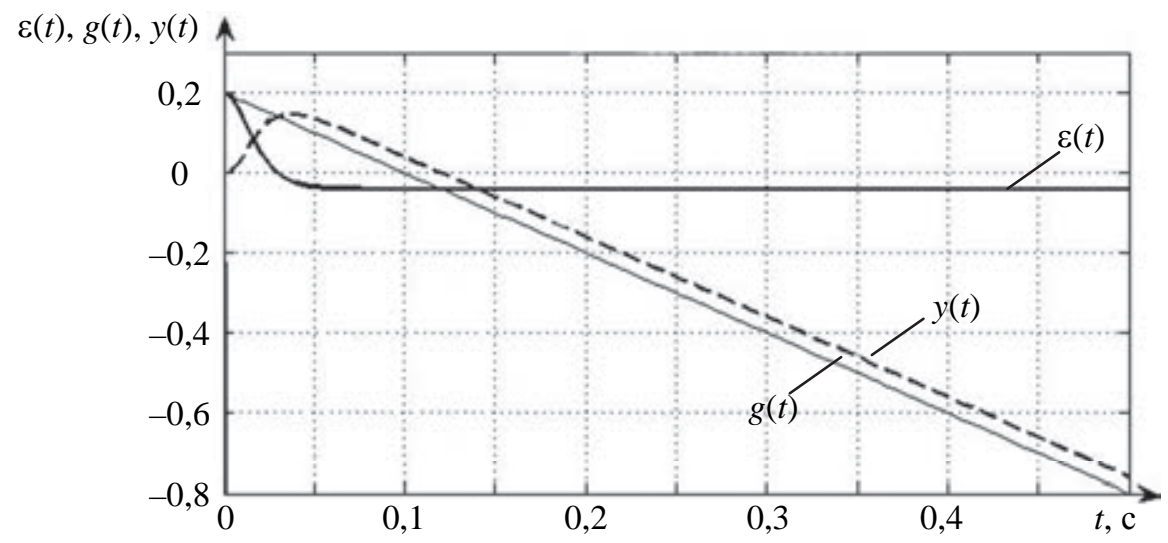

Рис. 5. Кривые внешнего воздействия, выхода и ошибки следящей системы с последовательным компенсатором, включенным по модифицированной схеме Смита

Дополнительно компьютерное исследование проведено для следящей системы с разработанным последовательным компенсатором, включенным в ее состав по схеме рис. 2. Для рассматриваемого случая соотношение (10) приняло вид $\tau \omega_{0}=3,729$, при этом для устойчивости системы должно выполняться неравенство $\tau \omega_{0}<3,729$. Для приведенных выше трех значений запаздывания $\tau=0,06 \mathrm{c}, \tau=0,3 \mathrm{c}, \tau=1,5 \mathrm{c}$ и вычисленного значения характеристической частоты $\omega_{0}=150 \mathrm{c}^{-1}$ указанное неравенство не выполняется. Действительно, 0,06.150=9>3,729; 0,3.150=45>3,729; $1,5 \cdot 150=225>3,729$. На рис. 6 приведены кривые выхода, ошибки и задающего воздействия $g(t)$ того 
же вида, что выше, для случая $\tau=0,06 \mathrm{c}$ и $\omega_{0}=150 \mathrm{c}^{-1}$. Нетрудно видеть, что процессы в системе вида рис. 2 расходятся как по выходу, так и по ошибке.

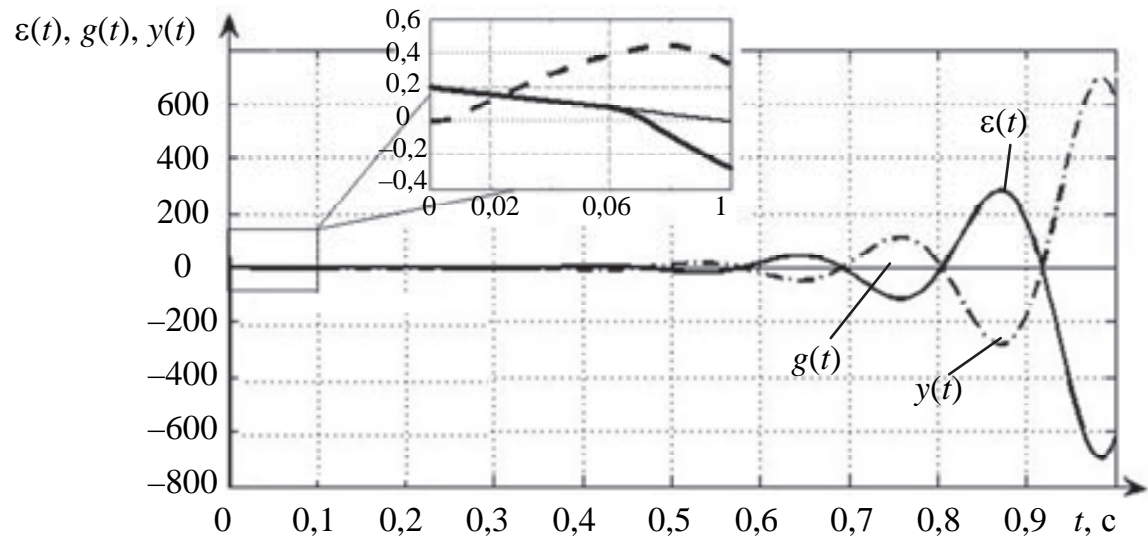

Рис. 6. Кривые задающего воздействия, выхода и ошибки в следящей системе рис. 2

10. В силу удовлетворения показателей качества спроектированной следящей системы сформулированным требованиям п. 1 можно осуществить переход к цифровой реализации последовательного компенсатора с помощью микроконтроллера, который должен функционировать с интервалом $\Delta t$ дискретности, при $\delta_{y}=0,05$ удовлетворяющим условию

$$
\Delta t \leq \frac{\pi}{\left(\frac{v_{n}}{\delta_{y}}\right)^{1 / n} \omega_{0}}=\frac{\pi}{\left(\frac{1}{0,05}\right)^{1 / 3} 150}=0,0078 \mathrm{c} .
$$

\section{Заключение}

В работе решена задача исключения влияния запаздывания в цепи основной обратной связи следящей системы с помощью последовательного компенсатора, включенного по схеме Смита. Для решения задачи исключения влияния запаздывания в цепи основной обратной связи следящей системы пришлось модифицировать базовую схему Смита включения последовательного компенсатора. Полученные теоретические результаты подтверждены комплексным компьютерным исследованием следящей системы с разработанным последовательным компенсатором, включенным в нее по модифицированной схеме Смита для случая объекта третьего порядка. Комплексное исследование показало, что процессы в спроектированной следящей системе нечувствительны к фиксированным значениям запаздывания в основной обратной связи.

\section{Литература}

1. Ким Д.П. Алгебраический метод синтеза систем управления с чистым запаздыванием // Мехатроника, автоматизация, управление. 2012. № 10. С. 2-7.

2. Гайдук А.Р. Теория и методы аналитического синтеза систем автоматического управления (полиномиальный подход). М.: Физматлит, 2012. 360 с.

3. Быстров С.В., Вундер Н.А., Синетова М.М., Ушаков А.В. Аналитическое конструирование последовательного компенсатора для систем с запаздыванием на основе модификации типовых полиномиальных моделей // Труды СПИИРАН. 2017. Т. 52 . № 3. С. 115-136. doi: $10.15622 /$ sp.52.6

4. Smith O.J.M. Close control of loops with dead time // Chemical Engineering Progress. 1957. V. 53. P. 217-235.

5. Smith O.J.M. A controller to overcome dead time // ISA J. 1959. V. 6. N 2. P. 28-32.

6. Majhi S., Atherton D.P. New Smith predictor and controller for unstable and integrating processes with time delay // Proc. $37^{\text {th }}$ IEEE Conference on Decision and Control. Tampa, USA, 1998. V. 2. P. $1341-1345$.

7. Palmor Z.J. Time-delay compensation - Smith predictor and its modifications / In: The Control Handbook. Ed. W.S. Levine. Boca Raton, USA, CRSC Press, 1996. P. 224-237.

8. Astrom K.J., Hang C.C., Lim B.C. A new Smith predictor for controlling a process with an integrator and long dead-time //

\section{References}

1. Kim D.P. Algebraic method of the synthesis control system for a plant with a pure (transport) delay. Mechatronics, Automation, Control, 2012, no. 10, pp. 2-7. (In Russian)

2. Gaiduk A.R. Theory and Methods of Analytical Synthesis of Automatic Control Systems (Polynomial Approach). Moscow, Fizmatlit Publ., 2012, 360 p. (In Russian)

3. Bystrov S.V., Vunder N.A., Sinetova M.M., Ushakov A.V. Analytical design of consecutive compensator for control systems with delay based on modification of typical polynomial models. SPIIRAS Proceedings, 2017, vol. 52, no. 3, pp. 115-136. (In Russian)

4. Smith O.J.M. Close control of loops with dead time. Chemical Engineering Progress, 1957, vol. 53, pp. 217-235.

5. Smith O.J.M. A controller to overcome dead time. ISA J, 1959, vol. 6, no. 2, pp. 28-32.

6. Majhi S., Atherton D.P. New Smith predictor and controller for unstable and integrating processes with time delay. Proc. $37^{\text {th }}$ IEEE Conference on Decision and Control. Tampa, USA, 1998, vol. 2, pp. 1341-1345.

7. Palmor Z.J. Time-delay compensation - Smith predictor and its modifications. In The Control Handbook. Ed. W.S. Levine. Boca Raton, USA, CRSC Press, 1996, pp. 224-237.

8. Astrom K.J., Hang C.C., Lim B.C. A new Smith predictor for controlling a process with an integrator and long dead-time. IEEE Transactions on Automatic Control, 1994, vol. 39, no. 2, 
IEEE Transactions on Automatic Control. 1994. V. 39. N 2. P. 343-345. doi: 10.1109/9.272329

9. Matausek M.R., Ribic A.I. Control of stable, integrating and unstable processes by the Modified Smith Predictor // Journal of Process Control. 2012. V. 22. N 1. P. 338-343. doi: 10.1016/j.jprocont.2011.08.006

10. Karafyllis I., Krstic M. Delay-robustness of linear predictor feedback without restriction on delay rate // Automatica. 2013.
V. 49 .
N 6.
P. 1761-1767.
doi: 10.1016/j.automatica.2013.02.019

11. Vunder N.A., Ushakov A.V. Sensitivity analysis of systems with a cascade compensator embedded in a Smith predictor to dead-time variation // Optoelectronics, Instrumentation and Data Processing. 2016. V. 52. N $3 . \quad$ P. 274-279. doi: 10.3103/S8756699016030092

12. Вундер Н.А., Ушаков А.В. Анализ чувствительности к вариации запаздывания систем с последовательным компенсатором, включенным по схеме Смита // Автометрия. 2016. № $3 . \quad$ С. 71-78. doi: 10.15372/AUT20160309

13. Быстров С.В., Вундер Н.А., Ушаков А.В. Решение проблемы сигнальной неопределенности при аналитическом конструировании последовательного компенсатора в задаче управления пьезоприводом // Научно-технический вестник информационных технологий, механики и оптики. 2016. Т. 16. № 3. С. 451-459. doi: 10.17586/2226-1494-2016-16-3-451-459

14. Mason S.J. Feedback theory - further properties of signal flow graphs // Proceedings of the IRE. 1956. V. 44. N 7. P. 920-926. doi: 10.1109/JRPROC.1956.275147

15. Zadeh L.A., Desoer C.A. Linear System Theory: The State Space Approach. NY: Dover Publications, 2008. 656 p.

\section{Авторы}

Быстров Сергей Владимирович - кандидат технических наук, доцент, доцент, Университет ИТМО, Санкт-Петербург, 197101, Российская Федерация, Scopus ID: 57195218699, ORCID ID: 0000-0002-6900-0506, sbystrov@mail.ru

Вундер Нина Александровна - аспирант, Университет ИТМО, Санкт-Петербург, 197101, Российская Федерация, Scopus ID: 57151208300, ORCID ID: 0000-0003-1201-4816, polinova_nina@mail.ru

Павлов Андрей Сергеевич - аспирант, Университет ИТМО, Санкт-Петербург, 197101, Российская Федерация, ORCID ID: 0000-0003-1154-9886, a.s.pavlov@email.su

Синетова Мадина Мансуровна - аспирант, Университет ИТМО, Санкт-Петербург, 197101, Российская Федерация, Scopus ID: 57195221561, ORCID ID: 0000-0002-5069-5053, sinetovamadina@gmail.com

Уиаков Анатолий Владимирович - доктор технических наук, профессор, профессор, Университет ИТМО, Санкт-Петербург, 197101, Российская Федерация, Scopus ID: 7103199773, ORCID ID: 0000-0001-5735-5249, ushakov-avg@yandex.ru pp. 343-345. doi: 10.1109/9.272329

9. Matausek M.R., Ribic A.I. Control of stable, integrating and unstable processes by the Modified Smith Predictor. Journal of Process Control, 2012, vol. 22, no. 1, pp. 338-343. doi: 10.1016/j.jprocont.2011.08.006

10. Karafyllis I., Krstic M. Delay-robustness of linear predictor feedback without restriction on delay rate. Automatica, 2013, vol. 49, no. 6, pp. 1761-1767. doi: 10.1016/j.automatica.2013.02.019

11. Vunder N.A., Ushakov A.V. Sensitivity analysis of systems with a cascade compensator embedded in a Smith predictor to dead-time variation. Optoelectronics, Instrumentation and Data Processing, 2016, vol. 52, no. 3, pp. 274-279. doi: 10.3103/S8756699016030092

12. Vunder N.A., Ushakov A.V. Sensitivity analysis of systems with a cascade compensator embedded in a Smith predictor to dead-time variation. Optoelectronics, Instrumentation and Data Processing, 2016, vol. 52, no. 3, pp. 274-279. doi: 10.3103/S8756699016030092

13. Bystrov S.V., Vunder N.A., Ushakov A.V. Solution of signal uncertainty problem at analytical design of consecutive compensator in piezo actuator control. Scientific and Technical Journal of Information Technologies, Mechanics and Optics, 2016, vol. 16, no. 3, pp. 451-459. (In Russian) doi: 10.17586/2226-1494-2016-16-3-451-459

14. Mason S.J. Feedback theory - further properties of signal flow graphs. Proceedings of the IRE, 1956, vol. 44, no. 7, pp. 920-926. doi: 10.1109/JRPROC.1956.275147

15. Zadeh L.A., Desoer C.A. Linear System Theory: The State Space Approach. NY, Dover Publications, 2008, 656 p.

\section{Authors}

Sergey V. Bystrov - PhD, Associate Professor, Associate Professor, ITMO University, Saint Petersburg, 197101, Russian Federation, Scopus ID: 57195218699, ORCID ID: 0000-00026900-0506, sbystrov@mail.ru

Nina A. Vunder - postgraduate, ITMO University, Saint Petersburg, 197101, Russian Federation, Scopus ID: 57151208300, ORCID ID: 0000-0003-1201-4816, polinova_nina@mail.ru

Andrey S. Pavlov - postgraduate, ITMO University, Saint Petersburg, 197101, Russian Federation, ORCID ID: 0000-00031154-9886, a.s.pavlov@email.su

Madina M. Sinetova - postgraduate, ITMO University, Saint Petersburg, 197101, Russian Federation, Scopus ID: 57195221561, ORCID ID: 0000-0002-5069-5053, sinetovamadina@gmail.com

Anatoly V. Ushakov - D.Sc., Full Professor, ITMO University, Saint Petersburg, 197101, Russian Federation, Scopus ID: 57150444100, ORCID ID: 0000-0001-5735-5249, ushakovavg@yandex.ru 